\title{
On the Dark Matter Halos of Optical and IR-selected AGN in the Local Universe
}

\author{
Mehmet Alpaslan, Jeremy L. Tinker \\ Center for Cosmology and Particle Physics, Department of Physics, New York University, New York, NY, 10003, USA
}

13 August 2020

\begin{abstract}
We use the technique of total satellite luminosity, $L_{\text {sat }}$, to probe the dark matter halos around active galactic nuclei in the SDSS Main Galaxy Sample. Our results focus on galaxies and AGN that are the central galaxy of their halo. Our two AGN samples are constructed from optical emission-line diagnostics and from WISE infrared colors. Both optically-selected and WISE-selected AGN have $L_{\text {sat }}$ values twice as high as non-active galaxy samples when controlling for stellar mass and mean stellar age. This implies that the halos are twice as massive, but we cannot rule out that the increase in $L_{\text {sat }}$ is due to these AGN residing in younger halos at the same mass. When only controlling for host galaxy stellar mass, WISE-selected AGN also have higher $L_{\text {sat }}$ values than optical AGN at the factor of two level, consistent with previous results comparing the clustering of obscured and unobscured AGN. However, controlling for stellar age in the two populations of host galaxies removes half of this difference, attenuating the statistical significance of the difference. We perform permutation tests to quantify the different in the halo populations of each sample. The difference in star formation properties does not fully explain the difference in the two AGN populations, however. Although AGN luminosity correlates with mean stellar age, the difference in stellar age between the WISE and optical samples cannot be fully explained by differences in their AGN luminosity distributions.
\end{abstract}

Key words: galaxies: haloes — galaxies: active

\section{INTRODUCTION}

Since the discovery of active galactic nuclei (AGN), the myriad classifications of AGN have expanded as data from disparate wavelengths have become available. But as the taxonomy of observational categories has expanded, progress has been made in unifying these varying observable properties into physical models of AGN activity (e.g., Antonucci 1993; Netzer 2015). The unified model of AGN posits the existence of a dusty torus around the supermassive black hole, the presence of which makes the observed properties of an AGN highly dependent on the inclination angle of the observation, even though the physical properties are the same. This model provides a natural explanation for two populations of AGN, one that exhibits minimal dust attenuation and is thus detected through optical data, and one of highly obscured systems that are identifiable at infrared (IR) wavelengths.

The advent of large IR surveys, most notably the WideField Infrared Survey Explorer (WISE; Wright et al. 2010), has created statistical samples of dust-obscured AGN that presents a complementary test of the unified model. If obscured and unobscured AGN only differ in their inclination with respect to the observer, then these two samples of objects should exhibit identical spatial clustering, implying they occupy the same set of dark matter halos. Although not all clustering studies agree, most have found a statically significant difference in the $\operatorname{bias}^{1} b$, of dust-obscured and unobscured AGN samples such that obscured AGN have higher clustering and thus occupy higher masses dark mat-

1 We define bias as $b^{2}=\xi / \xi_{m}$, where $\xi$ is the autocorrelation of the sample and $\xi_{m}$ is the correlation function of matter distribution. When measured at large scales, $r \gtrsim 10 h^{-1} \mathrm{Mpc}, b$ can be converted to halo mass assuming linear bias and a model of how linear bias scales with $M_{h}$ (e.g., Sheth \& Tormen 1999; Tinker et al. 2010; McClintock et al. 2019). Also when measured at large scales, this $b$ is equivalent to that measured using crosscorrelations of the tracer sample with the matter instead of autocorrelations. 
ter halos (Hickox et al. 2011; Donoso et al. 2014; DiPompeo et al. 2014, 2016; Mendez et al. 2016; DiPompeo et al. 2017). DiPompeo et al. (2017) used WISE data and Planck CMB data to measure both quasar clustering and CMB lensing by quasars. They found that obscured AGN have higher clustering than unobscured at the $4 \sigma$ level, yielding a factor of three difference in the inferred halo masses.

Mendez et al. (2016) used PRIMUS and DEEP2 data at $z \sim 0.7$, and thus spectroscopic redshifts, to analyze a smaller sample of AGN at lower luminosities. Mendez et al. (2016) found no statistical difference in the clustering of obscured and unobscured AGN, albeit with errors larger than the stated difference in halo masses found by DiPompeo et al. (2017). Due to the lower AGN luminosity, Mendez et al. (2016) was able to control for sample selection effects that plague comparisons between different AGN classes. More generally, their bias differences for samples of $\mathrm{X}$-Ray, IR, and radio-selected AGN were consistent with the differences in the host galaxy stellar masses and starformation properties.

In the local universe, clustering studies of X-ray selected AGN have produced conflicting results. Cappelluti et al. (2010) measured AGN autocorrelations, finding a lower clustering amplitude for obscured sources relative to unobscured. However, Krumpe et al. (2018), cross-correlating AGN sources with 2MASS galaxies, find no evidence for a difference in large-scale clustering amplitude. They did find that the overall AGN sample was less clustered than the reference galaxy sample, but this sample did not control for selection effects. Li et al. (2006) cross-correlated AGN identified from SDSS spectra with reference SDSS galaxy catalogs. These samples did control for AGN selection effects, and still found that AGN were less clustered than the general galaxy population.

In this paper, we present a probe of dark matter halos around AGN that is complementary to the clustering tests. Using a sample of central galaxies ${ }^{2}$ in the Main Galaxy Survey (MGS) of the Sloan Digital Sky Survey (Strauss et al. 2002), Tinker et al. (2019a) and Alpaslan \& Tinker (2019) cross-correlated the spectroscopic MGS central galaxies with faint imaging data to measure the total amount of luminosity in satellite galaxies around them. Hierarchical clustering in $\Lambda \mathrm{CDM}$, combined with simple prescriptions for mapping galaxies onto dark matter halos, predicts that this quantity, $L_{\text {sat }}$, should scale strongly with host halo mass (Tinker et al. 2019a). Although it is not a direct observable of the gravitational potential around a galaxy, like weak lensing or satellite kinematics, $L_{\mathrm{sat}}$ is an indirect probe of the halo that affords a much higher signal-to-noise measurement than direct observables. Thus this quantity is more effective with small samples of galaxies, such as AGN.

$L_{\text {sat }}$ is complementary to clustering in multiple ways. First, it is simply an independent quantity uncorrelated with the large-scale two-point correlation function. By the nature of our sample construction, we only analyze central galaxies, removing satellite galaxies from consideration. This makes

2 We define a central galaxy as the galaxy located at the center of the host dark matter halo. Satellite galaxies are those located within the host halo but not at the center. A host halo is one that is not located within the virial radius of any other halo. the interpretation of the inferred halo masses more clear. Second, $L_{\text {sat }}$ breaks degeneracies with halo properties that affect clustering apart from halo mass. The implicit assumption in the clustering studies cited above is that the connection between AGN properties and dark matter halos is confined only to the mass of the halo-i.e., more massive halos cluster more strongly, thus differences in $b$ imply differences in $M_{h}$. However, it is well-known that halo clustering is highly dependent on other properties apart from mass. This effect is referred to as 'halo assembly bias (see, e.g., the review by Wechsler \& Tinker 2018 and references therein). For example, at fixed $M_{h}$, early-forming halos show significantly enhanced clustering relative to their late-forming counterparts. Thus, the enhanced clustering of dust-obscured AGN may be driven by the formation history of the halos they occupy, rather than the halo mass. This would still support the interpretation that dust-obscured AGN are distinct from optical AGN, but the connection with the dark sector-and thus possible evolutionary scenarios - is markedly different.

The connection to $L_{\text {sat }}$ is that, in addition to impacting clustering, early-forming halos have significantly less substructure as well. For example, if the elevated bias in DiPompeo et al. (2017) is to due assembly bias, $L_{\text {sat }}$ for dust-obscured AGN would be suppressed relative to optical samples, and suppressed relative to the overall sample of non-AGN galaxies. On the other hand, if the enhanced clustering is truly due to the halo mass, the $L_{\text {sat }}$ would be enhanced as well, by roughly the same factor as the $M_{h}$.

We will show $L_{\text {sat }}$ results for three different samples of central galaxies from the MGS: the overall sample, which we will use as a control sample, a sample of optical AGN within the MGS selected using spectral line diagnostics, and a sample of dusty AGN selected from the MGS using broadband colors from WISE data. Like Mendez et al. (2016), we will control for sample selection effects the estimated halo masses. In $\S 2$ we present our data, including the sample of central galaxies, with relevant background on how we measure $L_{\text {sat }}$. In $\S 3$ we present the $L_{\text {sat }}$ results for the different samples of galaxies. Due to the limited size of these samples, we focus on the statistical significance of the result, presenting multiple ways to analyze the results. In $\S 4$ we will compare our results to previous data and discuss them in the context of the unified AGN model. For all distance calculations we assume a flat, $\Lambda \mathrm{CDM}$ cosmology with $\Omega_{m}=0.3$.

\section{DATA}

This analysis is based on the sample of MGS central galaxies and $L_{\text {sat }}$ measurements from Alpaslan \& Tinker (2019). This sample is constructed from the NYU Value-Added Galaxy Catalog (NYU-VAGC; Blanton et al. 2005). Specifically, we use the magnitudes, redshifts, and positions from the dr72bright34 sample. Derived galaxy quantities, specifically $D_{n} 4000$ and AGN classification, are obtained from the MPA-JHU value-added catalog ${ }^{3}$. The MPA-JHU catalog corresponds to the DR8 SDSS public data release, which contains all galaxies in the DR7 sample. Additional photometric data is from the DECALS Legact Imaging Surveys

\footnotetext{
${ }^{3}$ https://www.sdss3.org/dr10/spectro/galaxy_mpajhu.php
} 
(DLIS; Dey et al. 2019), which is a combination of DECam data at declination $\leqslant 30^{\circ}$ and Kitt Peak data at higher declination. Although the current DLIS data release is DR8 (NB, this numbering scheme is distinct from the SDSS DR numbers), the Alpaslan \& Tinker (2019) results use DR6 (from Kitt Peak) and DR7 (from DECam) results. The DR6+DR7 combined photometry covers roughly $75 \%$ of the final MGS footprint. We detail our samples, and how these data are used, further in this section.

\subsection{Total Satellite Luminosity}

Total satellite luminosity is a method for probing the relationship between galaxies and halos. The amount of substructure within a dark matter halo is roughly self-similar with the mass of the halo. These substructures will contain faint satellite galaxies, thus the total amount of light in satellite galaxies is a strong probe of the dark matter halo around the galaxy that sits at the center of that halo. Most satellite galaxies are below the magnitude limit of the MGS spectroscopic sample, thus the $L_{\text {sat }}$ measurements require using deeper imaging data. In Tinker et al. (2019b), we used DLIS data as our source of imaging due to its increased depth and better resolution over legacy SDSS imaging. The typical $r$-band depth of the DLIS data is $r \approx 24$, as compared to the MGS spectroscopic limit of $r=17.7$. To measure $L_{\text {sat }}$, we sum the total $r$-band light from imaging galaxies, excluding the spectroscopic central galaxy, within a $50 h^{-1} \mathrm{kpc}$ (comoving) aperture. To estimate what fraction of those galaxies are associated with the halo, as opposed to random interlopers projected along the line of sight, we measure the expected interloper contribution from randomly placed apertures of the same size. For both central galaxies and randomly placed apertures, we require that there are no other MGS spectroscopic galaxies within the aperture. After subtracting off the background contribution for a given central galaxy, we calculate luminosity by assuming that all objects are at the redshift of the central galaxy. We limit the $L_{\text {sat }}$ value to satellites brighter than $M_{r}-5 \log h=-14$. We refer the interested reader to Tinker et al. (2019a) for a comprehensive description of the measurements and tests of the method.

Measurements of $L_{\text {sat }}$ for a single galaxy are, of course, too noisy to be of much use. A significant fraction of galaxies have negative values of $L_{\text {sat }}$ simply due to random variation in the background galaxy counts, as well as fluctuations in the intrinsic number of satellites in a give halo. Thus, we stack central galaxies and take the average $L_{\text {sat }}$ values. In Alpaslan \& Tinker (2019), we demonstrated that $L_{\text {sat }}$ correlates strongly with $M_{*}$, as expected from the correlation between $M_{*}$ and $M_{h}$. At fixed $M_{*}, L_{\text {sat }}$ also correlated with other galaxy properties, implying that these properties carry information about the halos they live in, beyond the information encoded in their stellar masses. Alpaslan \& Tinker (2019) used additional data - the large-scale density around the satellite galaxies - to break this degeneracy between $M_{h}$ and halo assembly bias. Here, due to lack of statistics to probe environments, we focus on the $L_{\text {sat }}$ measurements themselves as a test of whether the halo around different types of AGN are distinct from each other and distinct from the overall non-AGN population.

\subsection{Central Galaxies}

To characterize MGS galaxies we use stellar masses using the PCA method of Chen et al. (2012). This is the same stellar mass definition employed in Tinker et al. (2019a) and Alpaslan \& Tinker (2019). All analyses in this paper are performed on galaxies that are inferred to be central within their dark matter halo. Although there is a population of AGN within the set of satellite galaxies (Pasquali et al. 2009), $L_{\text {sat }}$ measurements are much more difficult to interpret for satellites, as the number of faint galaxies around a satellite is more a function of host halo and the satellite's position within it. For central galaxies, $L_{\text {sat }}$ is direct probe of the host dark matter halo.

Methods to determine whether or not a galaxy is a central will always yield a sample with some amount of incompleteness and impurities. Group catalogs on volume-limited samples can construct sets of central galaxies with both high completeness and purity (Tinker et al. 2018), but requiring that the galaxy sample be volume-limited is highly restrictive. Given the low frequency of AGN, being able to analyze the largest possible parent sample of galaxies is paramount. In this paper we use the 'central galaxy finder' introduced in Tinker et al. (2019a), and used in Alpaslan \& Tinker (2019). In tests using a mock flux-limited MGS-like sample, and using a restrictive criteria to minimize impurities, this method yields a sample of central galaxies that is $93 \%$ pure and $72 \%$ complete. The small amount of satellite galaxies that make it into this 'pure' central sample do impact the average $L_{\text {sat }}$ measured in bins of central galaxy stellar mass; at $M_{*} \lesssim 10^{10.5} \mathrm{M}_{\odot}$, impurities increase $L_{\text {sat }}$ by $0.1-0.2 \mathrm{dex}$, while for more massive galaxies the impacts are nearly negligible. Because AGN have roughly the same satellite fraction as the overall galaxy population (Pasquali et al. 2009), this should not bias the comparison of $L_{\text {sat }}$ between AGN samples and non-AGN samples.

\subsection{AGN Selection}

Optically-selected AGN are taken from the public MPAJHU DR8 catalog. The selection is described in Brinchmann et al. (2004) and Kauffmann et al. (2003). It is based on the BPT (Baldwin et al. 1981) line-ratio diagnostic to separate star-forming and active galaxies. The sample we use is a strict AGN classification, removing galaxies with line ratios indicative of LINERS and composite spectra. From 559,028 galaxies in the NYU-VAGC sample, 17,272 of them match this classification. After restricting the sample to be only 'pure' central galaxies, removing objects that have MGS interlopers along the line of sight, and choosing only galaxies that lie in the overlap with DLIS DR6+DR7 imaging, the number of optical AGN used in the analysis is 4,432.

Infrared-selected AGN are identified through the use of WISE photometry available in the DLIS public data release (Dey et al. 2019). Many studies have shown WISE colorcolor analysis can effectively isolate dust-obscured AGN from the general galaxy population (Jarrett et al. 2011; Yan et al. 2013; Satyapal et al. 2014) In this work, we use the unWISE co-added data, with forced photometry for DLIS optical sources, from Lang (2014). All MGS objects are detected in the DLIS imaging where the two surveys overlap, and matched catalogs between the DLIS data releases and 


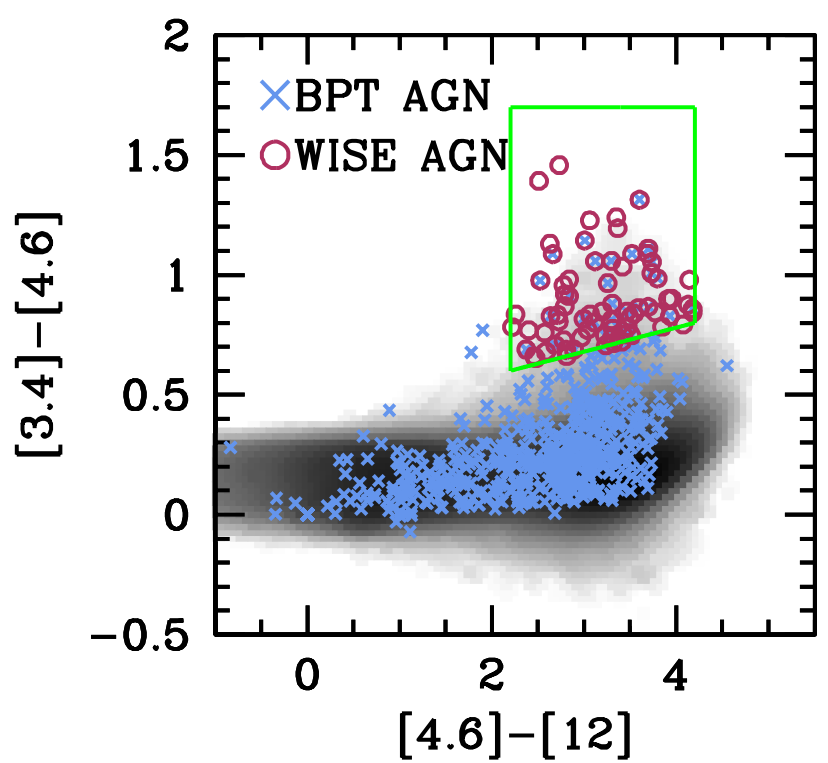

Figure 1. Selection of dust-obscured AGN in WISE color-color space. The axis labels indicate the WISE bands used in each color. The grayscale represents the number density of MGS galaxies at each point in color space, using a logarithmic color scale. The blue crosses show a random sample of galaxies that are identified as AGN using the BPT line diagnostics in the MPA-JHU catalog. The red circles are objects contained within the green trapezium in the upper right. These frequency of these objects is $3.1 \%$ of the overall sample. These color cuts are taken from Jarrett et al. 2011, optimized to find dust-obscured AGN. The frequency of these objects is $0.8 \%$ of the full sample.

the SDSS Legacy samples are provided on the DLIS public pages ${ }^{4}$. Figure 1 shows a subsample of MGS galaxies in WISE color-color space. Those objects that are also identified as optical AGN using the line diagnostics are also indicated. The green trapezium shows the AGN selection from Jarrett et al. (2011). In the NYU-VAGC, 1,942 objects pass this selection. After trimming the catalog in the same manner as for the BPT selection, the final number of WISE AGN used in the analysis is 449 .

\section{$2.4 \quad$ Host galaxy properties}

The host galaxy properties of these two AGN samples, relative to the overall sample of MGS central galaxies, is shown in Figure 2. To characterize these populations, we use galaxy stellar mass, the broadband $g-r$ color, and the $4000-\AA$ break in the galaxy spectra, $D_{n} 4000$. The $g-r$ color is sensitive to both the age of the stellar population and the amount of dust in the host galaxy. A value of $g-r=0.8$ is a rough break-point between the blue cloud and red sequence. The $4000-\AA$ break is also sensitive to the mean stellar age of the host galaxy, but is largely insensitive dust content, making it a more efficient diagnostic for identifying star-forming and quiescent galaxies. For the overall galaxy population, the distribution of $\mathrm{D}_{n} 4000$ is more clearly bimodal than the color distribution. A break-point of $\mathrm{D}_{n} 4000=1.6$, at the

${ }^{4}$ http://legacysurvey.org/ minimum between the two modes, is a reasonable separation point between star-forming and quiescent galaxy samples.

For both AGN samples the median stellar mass is $\sim 10^{10.8} \mathrm{M}_{\odot}$, with a dearth of both very massive and lowmass galaxies. The host galaxies of BPT-AGN have redder broadband colors than the overall sample, but the distribution is unimodal and peaks at the valley between the two modes in the MGS distribution. Additionally, the $\mathrm{D}_{n} 4000$ values also peak at the transition point between star-forming and quiescent populations, as shown by Kauffmann et al. (2003). The fraction of BPT host galaxies above the breakpoint in color is $52 \%$, while the fraction at $\mathrm{D}_{n} 4000>1.6$ is $47 \%$. The $g-r$ color distribution for WISE-AGN host galaxies is also unimodal, but the peak is shifted slightly blue-ward of the BPT distribution. The mean sellar ages of the WISE host galaxies, however, are clearly indicative of a sample of actively star-forming galaxies. The fraction of WISE-AGN host galaxies in the red sequence is $34 \%$, but the fraction categorized as quiescent by their $\mathrm{D}_{n} 4000$ break is only $15 \%$. This is consistent with the IR-selected AGN sample in Mendez et al. (2016), which were predominantly star-forming objects, as well as the $z \sim 0$ of WISE-selected AGN in Weston et al. (2017).

\section{RESULTS}

We are asking three distinct questions: Are the halos of WISE-AGN distinct from the overall non-AGN population? Are the halos of BPT-AGN also distinct from the overall galaxy population? And last, are the halos of the different AGN classes distinct from each other? We will investigate these questions in three different ways: by directly measuring the $L_{\mathrm{sat}}-M_{*}$ relations for the three different samples, by comparing the mean $L_{\text {sat }}$ of the AGN samples to the expected mean given the stellar mass distribution of the samples, and by use of a permutation test.

\subsection{Mean $L_{\text {sat }}$}

Figure 3 shows the mean $L_{\text {sat }}$ as a function of $M_{*}$, taken from Alpaslan \& Tinker (2019). In each bin, the error is calculated using 100 bootstrap resamples of the sample of central galaxies. The mean $L_{\text {sat }}$ for AGN identified in the BPT sample are shown with the blue symbols. The results are noisy at $M_{*} \lesssim 10^{10} \mathrm{M}_{\odot}$, but within the errors the $L_{\text {sat }}$ values for BPT AGN are either consistent with, or slightly higher than, the overall sample of central galaxies. In subsequent subsections we will make this comparison more quantitatively. The $L_{\text {sat }}$ values for the WISE-AGN, however, are clearly above the mean relation. The small sample size yields large statistical errors for the WISE-AGN sample. Thus, if one were to calculate these two sets of measurements using a $\chi^{2}$ statistic, one would find minimal difference between the two samples. However, it is worth noting that all the values of $L_{\text {sat }}$ for the WISE-AGN are above the mean relation. And if the measurements for this sample are merely statistical fluctuations, those fluctuations would need to go in the same direction for the vast majority of the sample. We present our statistical tests of this scenario presently. 

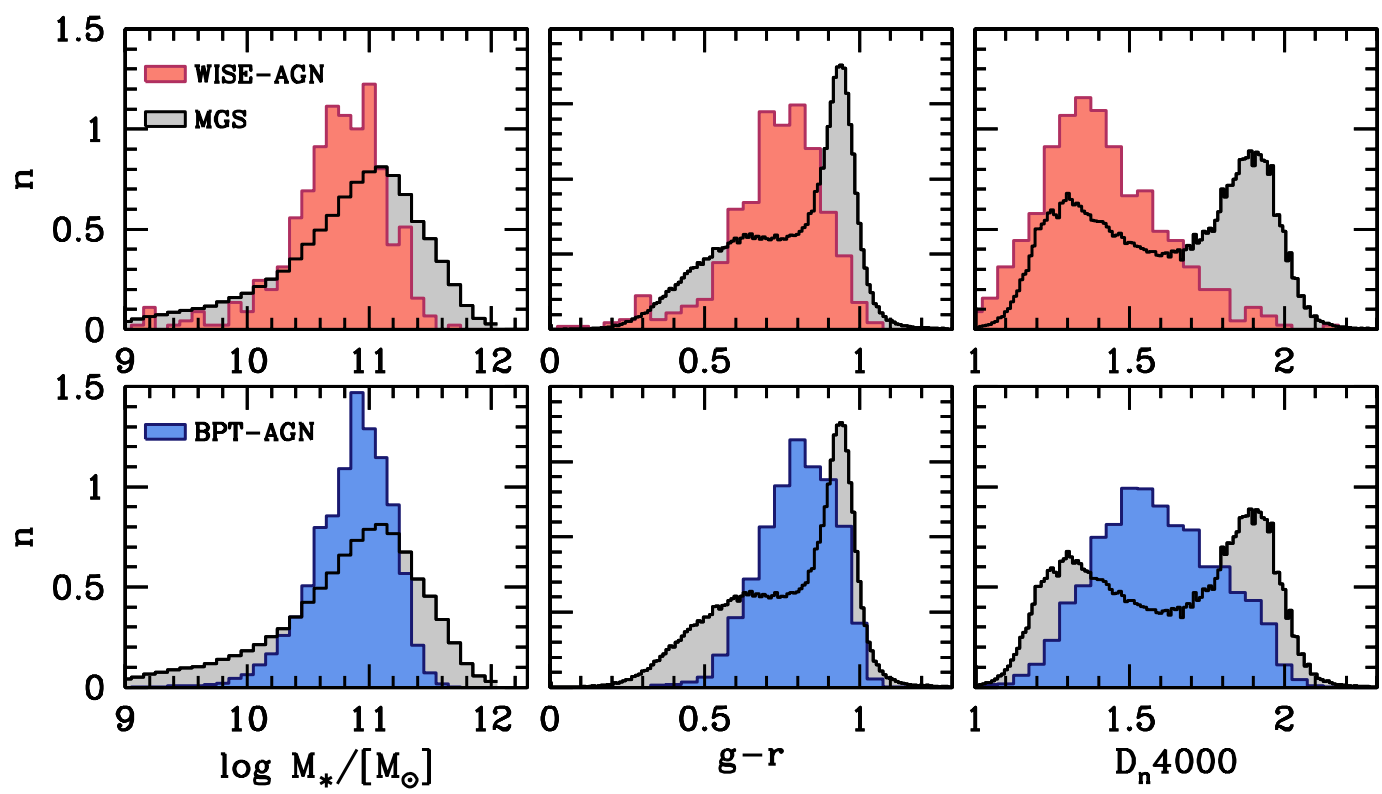

Figure 2. Properties of the AGN samples relative to the overall MGS sample of central galaxies. Top Row: Comparison of the normalized distributions, $n$, of stellar mass, $g-r$ color, and $D_{n} 4000$ for the WISE-selected sample. Bottom Row: Same as the top row, but now for the distribution of BPT-selected AGN.

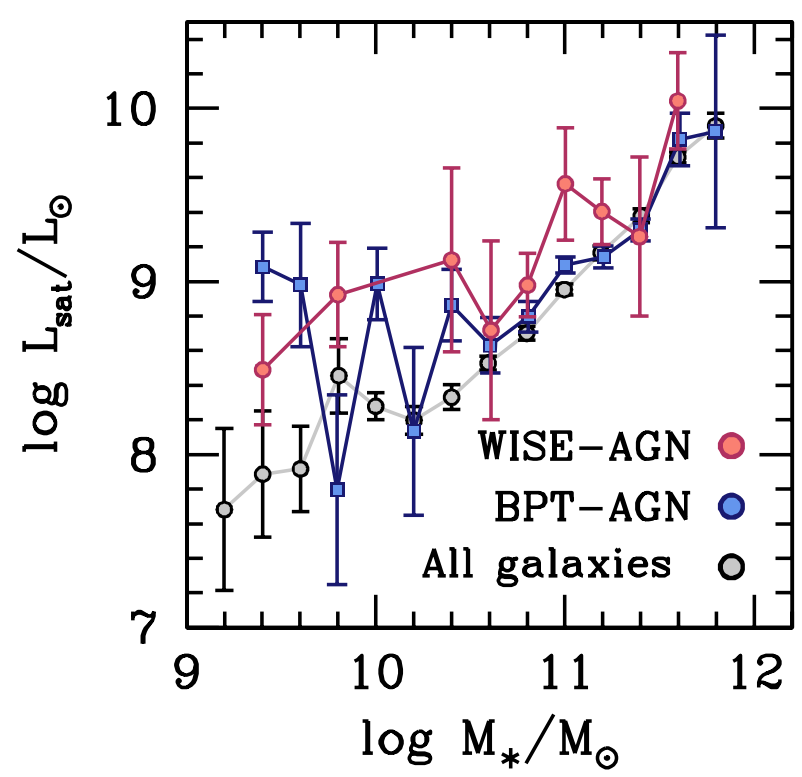

Figure 3. The mean $L_{\mathrm{sat}}$ as a function of central galaxy stellar mass, $M_{*}$. The gray connected circles show the relation for the full sample of central galaxies. The connected blue circles show the relation for the BPT-selected AGN sample. The connected red circles show the relation for the WISE-selected AGN sample. For each data point, the error bar is estimated using bootstrap resampling of the central galaxy samples.

\subsection{Are the $L_{\text {sat }}$ values different?}

Figure 4 shows our first quantitative test of the three populations of central galaxies. As demonstrated in Figure $3, L_{\text {sat }}$ depends strongly on $M_{*}$. However, binning the AGN sam- ples by $M_{*}$ only reduces the numbers of objects to work with, making it difficult to determine whether the AGN samples are above the mean. Thus, we define a quantity $\chi=L_{\text {sat }} / L_{\text {exp }}$, where $L_{\exp }$ is the "expected" value of $L_{\text {sat }}$ at a given value of $M_{*}$, given the mean relation in Figure 3. For the sample of BPT-AGN, the mean value is $\bar{\chi}=1.29 \pm 0.13$. For the sample of WISE-AGN, the mean value if $\bar{\chi}=2.42 \pm 0.64$. For both these measurements, the error in the mean is estimated by bootstrap resampling on the AGN sample. The bootstrap distributions of $\bar{\chi}$ for both BPT and WISE samples are shown in Figure 4. From the bootstrap analysis alone, WISE-selected AGN live in halos that have $L_{\text {sat }}$ values $1.88 \pm 0.52$ higher.

From the host galaxy properties in Figure 2, we note that $M_{*}$ is not the only galaxy parameter that may have an impact on $L_{\text {sat }}$ and the expected halo mass. Using weak lensing Mandelbaum et al. (2016) found strong bimodality in the halo halo masses of massive red and blue central galaxies, with red galaxies occupying higher mass halos. These results agree well with the $L_{\text {sat }}$ results in Tinker et al. (2019a) for red and blue subsamples, under the assumption that $L_{\text {sat }}$ is a function of $M_{h}$ only. Going beyond a simple red-blue split of the galaxy population, Alpaslan \& Tinker (2019) found that $L_{\text {sat }}$ correlated continuously with $\mathrm{D}_{n} 4000$ at fixed $M_{*}$, with a minimum at the green valley. Given that the peak of the $\mathrm{D}_{n} 4000$ distribution for BPT-AGN is in the green valley, we can ask what $\bar{\chi}$ is for a random sample of central galaxies with both the same $M_{*}$ and $\mathrm{D}_{n} 4000$ distributions.

The gray histograms in Figure 4 show the distribution of $\bar{\chi}$ values for random samples of the non-AGN galaxy population, selected such to match the distributions of $M_{*}$ and $\mathrm{D}_{n} 4000$, and matching the number of objects in each sample. For the random sample tailored to the WISE-selected sample, $\bar{\chi}$ is unbiased relative to the the general population. The results for the BPT-like random sample is quite differ- 

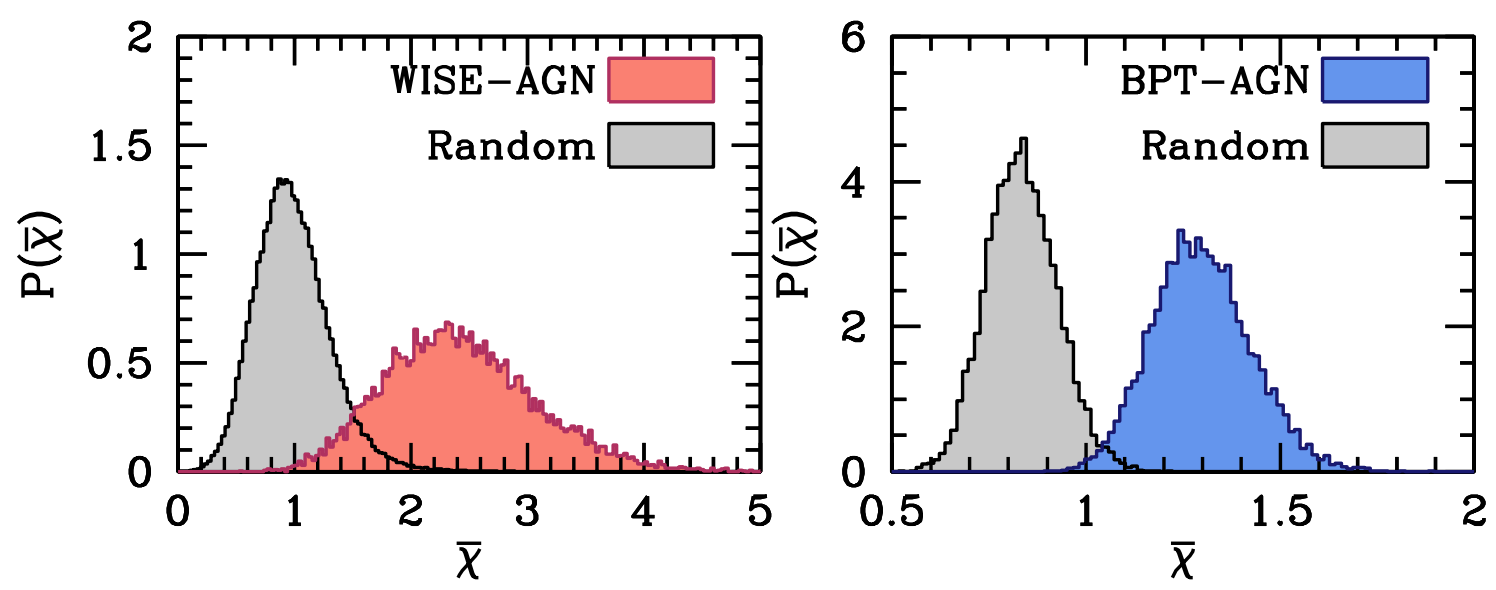

Figure 4. The probability distribution functions of the quantity $\bar{\chi}$, defined as the mean of $L_{\text {sat }} / L_{\text {exp }}$. In both panels, the gray histograms show the distributions of $\bar{\chi}$ for random samples of the population of non-AGN central galaxies, where each random sample has the same size as the AGN sample. The random selection is weights such that the distribution of $M_{*}$ in the AGN sample is matched. Note the difference in the $x$-axis ranges in both panels; because the BPT-AGN sample is $\sim 10$ times larger, the width of the $\bar{\chi}$ PDF is significantly smaller. In each panel, the colored histogram is the distribution of $\bar{\chi}$ from bootstrap resampling.

ent. Given their location in the green valley, $\bar{\chi}$ for the BPTmatched random sample is significantly lower than expected by $M_{*}$ alone, with a $\bar{\chi}=0.83 \pm 0.09$. Combining this with the bootstrap results of $\bar{\chi}$ for the BPT-AGN, these AGN have $L_{\text {sat }}$ values $1.54 \pm 0.16$ times higher than expected from given the demographics of their host galaxies.

Thus, when controlling for both stellar mass and star formation in the comparison of the $\bar{\chi}$ values for the WISE and BPT samples, the ratio of WISE/BPT is $1.57 \pm 0.44$. The WISE AGN live in halos with higher $L_{\text {sat }}$ values, but the statistical significance of this result is very low, at only $1 \sigma$.

\subsection{Permutation tests}

In order to assess the statistical significance of the differences between the $L_{\text {sat }}$ values of the different AGN populations, we perform a permutation test (also referred to as a randomization test). For two given samples of observations $A$ and $B$ with means $\bar{x}_{A}$ and $\bar{x}_{B}$ a permutation test can measure, by examining the difference between the two sample means, the statistical significance of the null hypothesis $H_{0}$ that both $A$ and $B$ are drawn from the same parent distribution. In practice, the test is simple to perform: the difference between both means, $T_{0}$, is computed. Following this initial calculation, all observations from $A$ and $B$ are pooled together and resampled at random into new subsamples according to the relative sizes of both samples $\left(N_{A}\right.$ and $\left.N_{B}\right)$. The difference of means is calculated again, and this procedure is repeated for every possible permutation of $A$ and $B$, yielding a distribution of the difference of means. Where $T_{0}$ lies relative to this distribution then allows one to accept or reject the null hypothesis. Our expectation is that the results of these tests should generally follow those from the previous subsection, but they are a quantitatively distinct test.

In our implementation of the permutation test, we set $N_{A}=N_{B}$ and, when comparing MGS galaxies to AGN samples, randomly sample the larger of the two populations such that the distribution of galaxy properties matches the sample selection of the smaller sample. We perform the test for a total of 1,000 random samples from the larger population, where the results have converged. Each test yields a deviate - a location of $T_{0}$, in the range of $[0,1]$, in the distribution created by the permutations. For example, a deviate of 0.5 is exactly at the median of the permutations, and a value of 0.99 means the null hypothesis can be excluded at $3 \sigma$ confidence.

In Figure 5 we show 'violin' plots of the results of performing permutation tests on the different AGN samples we have examined in this work. In the first two violins, we have sampled the the MGS galaxies to match the distribution of stellar masses of the AGN samples. The figure shows the distribution of deviates, with the central thick bar displaying the inner-50\% range; the horizontal black line displaying the median, and the full distribution being shown as the wings of the violins. The horizontal gray lines show the $1 \sigma-3 \sigma$ significance values. One final consideration is that for the purposes of our results, we can reject the null hypothesis if the statistical significance of the difference in the means is positive or negative. In other words, if the full distribution of the mean differences were normally distributed around 0 with a nonzero standard deviation, we can reject the null hypothesis if the difference between the two means were on the positive or negative tail of the distribution, so long as it was at or above $3 \sigma$ significance. For this reason, in Figure 5 we only show the deviates from 0.5 to 1 , and when performing the actual calculation we map all deviate values below 0.5 to be between 0.5 and 1 . We note that this only impacts the results for the BPT-MGS comparison.

We will discuss the permutation results presented in Figure 5 from left to right. For the BPT-MGS comparison, we cannot reject the null hypothesis that these two samples have the same distribution of $L_{\text {sat }}$ values, and thus the same dark matter halos. For the WISE-MGS comparison, on the other hand, we can reject the nll hypothesis. Greater than $75 \%$ of the deviates are above the $2 \sigma$ line. Additionally, but 


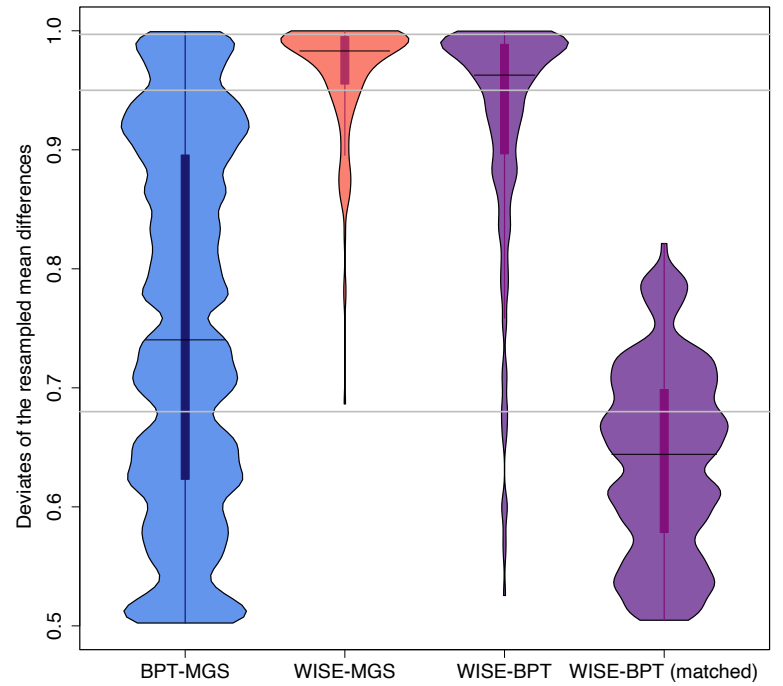

Figure 5. Violin plots showing the distributions of deviates generated in 1,000 realizations of the permutation test for all AGN samples. See text for details on our implementation of the test. For the first three violins, results only control for stellar mass in the comparison of the distributions. The grey horizontal lines denote quantiles that correspond to a $1 \sigma, 2 \sigma$, and $3 \sigma$ deviate respectively. These results suggest that there is an almost $3 \sigma$ significance in the permutation test result between WISE-selected and BPT-selected AGN, when controlling for stellar mass. The right violin (labeled as "matched"), however, shows how this result is mitigated when controlling for $\mathrm{D}_{n} 4000$ in the comparison as well.

with somewhat less confidence, we reject the null hypothesis that the WISE and BPT samples are drawn from the same distribution. These results are in agreement with the $\bar{\chi}$ results from Figure 4 . We note again that these first three results only control for stellar mass in the permutation tests. The last violin shows the WISE-BPT comparison, now controlling for both $M_{*}$ and $\mathrm{D}_{n} 4000$. Here, as in the previous subsection, the significance of the difference between the two samples is eliminated, implying that the difference in the two sets of halos is driven mostly by the star-forming properties of their host galaxies.

Figure 6 explores the BPT-MGS comparison in more detail. The first two violins compare the two samples, but for only quiescent and star-forming galaxies, respectively, from both samples. Here we use $\mathrm{D}_{n} 4000=1.6$ to separate the two samples. In contrast to the BPT-MGS comparison in FIgure 5 , we can reject the null hypothesis at $\sim 2 \sigma$ for each subsample. The right-most violin shows a full comparison, but now matching the distribution of both $M_{*}$ and $\mathrm{D}_{n} 4000$. In this comparison, we can reject the null hypothesis at nearly $3 \sigma$.

\section{SUMMARY AND DISCUSSION}

In this paper we have provided multiple ways to test whether optically-selected AGN and IR-selected AGN are part of the same overall population of objects, and whether either either

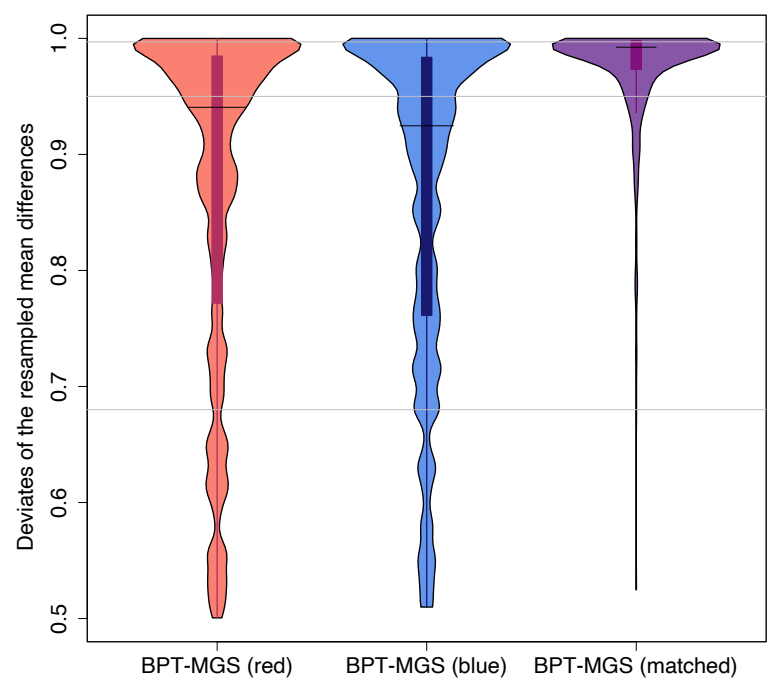

Figure 6. Violin plots for the comparison of the BPT-AGN sample to the non-AGN sample. From left to right, the three violins indicate the distribution of deviates for galaxies (and AGN host galaxies) classified as "red" (quiescent) by their $\mathrm{D}_{n} 4000$ value, galaxies classified as "blue" (star-forming) by $\mathrm{D}_{n} 4000$, and where the MGS population is sampled to match both the stellar masses and $\mathrm{D}_{n} 4000$ values of the BPT-AGN sample.

sample live in distinct halos from the general population of galaxies.

The $L_{\text {sat }}$ measurements demonstrate that both samples of AGN live in distinct halos from the general population when controlling for stellar mass and mean stellar age. For the $\bar{\chi}$ test, the WISE-selected AGN have higher $L_{\text {sat }}$ values at $\sim 2 \sigma$ level, while in the permutation test the WISE population of halos is distinct from a random sample at nearly $3 \sigma$ For the BPT-selected AGN, the $\bar{\chi}$ test finds that the halos of these AGN have higher $L_{\text {sat }}$ values at $3 \sigma$ significance, while the permutation test settles in to just under $2 \sigma$ confidence. We note that, when only controlling for stellar mass and not for $\mathrm{D}_{n} 4000$, the conclusions for the WISE-AGN sample are unchanged, but the evidence for BPT-AGN being in different halos would be substantially weaker.

The clustering study of $\mathrm{Li}$ et al. (2006) is closest to our $L_{\text {sat }}$ analysis in terms of sample selection and systematic controls. They compared the clustering of AGN in SDSS to samples of galaxies controlled by various properties, includ$\operatorname{ing} M_{*}$ and $\mathrm{D}_{n} 4000$. They found that AGN were less clustered than the control samples, more so when $\mathrm{D}_{n} 4000$ was used as part of the control sample. This is the opposite of the signal measured here. The AGN sample they used, however, is significantly different. They included LINERS and composite spectra, yielding a sample significantly larger than ours. If AGN selection is the cause of the difference, then the additional populations in their AGN would have to be in significantly lower mass halos than Seyfert objects. If sample selection is not the explanation, then galaxy assembly bias could reconcile these results - as discussed in the introduction, halos with more substructure tend to have weaker clustering at fixed $M_{h}$, implying that late-forming halos are 

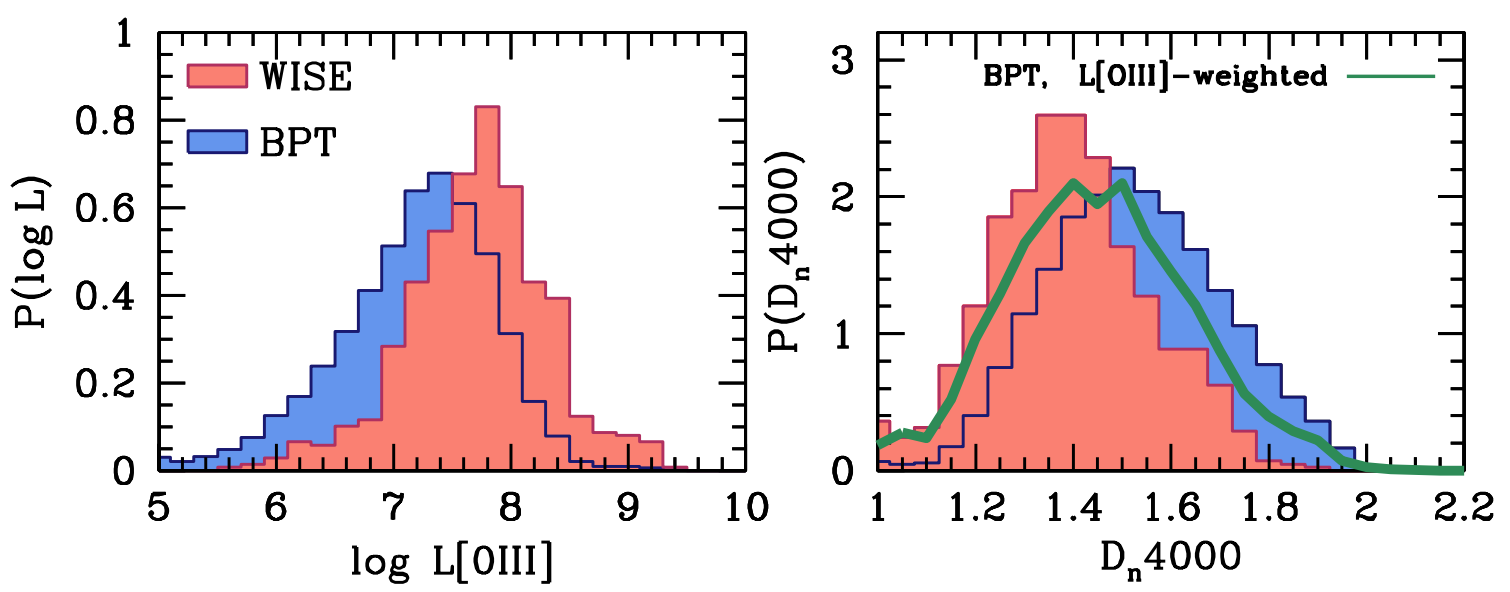

Figure 7. left Panel: Normalized distributions of $\log L_{[\mathrm{OIII}]}$ in MGS galaxies identified as AGN in their spectra. [OIII] luminosity values are given in units of solar bolometric luminosity, in the same manner as in Kauffmann et al. 2003. Right Panel: Normalized distributions of $\mathrm{D}_{n} 4000$ AGN. The blue histogram is all BPT AGN, while the red histogram are those AGN that lie within the WISE selection box in Figure 1. The dark green curve is the distribution of $\mathrm{D}_{n} 4000$ of the BPT sample, when weighted to match the $L_{[\mathrm{O} I I I]}$ distribution of WISE AGN. Although the mode of the weighted distribution is shifted to lower $\mathrm{D}_{n} 4000$, a KS test yields a probability that the red histogram is drawn from the green distribution at $10^{-21}$.

more likely to undergo AGN activity. Further study is required to reconcile these results.

A third method of obtaining halo massess - distinct from both clustering and $L_{\text {sat }}$-is galaxy group catalogs. Weston et al. (2017) use a similar WISE color selection to find dusty AGN in the SDSS MGS for the purpose of investigating merger activity. They obtain halo masses from the group catalog of Yang et al. (2007). When restricting to a sample of interacting and merging galaxies, the same of WISE AGN is only 18 systems. Within this sample, the median halo mass for central galaxies is 0.2 dex smaller than that of the non-AGN sample. However, given the limited sample size, a KS test yields a $P_{K S}$-value of 0.1 . Further, the Yang catalog - along with most other group-finding algorithms - use the total stellar mass of the group to match to halo mass. Thus, when the central galaxy dominates the stellar mass of the group - as it does at the mass scales probed here (e.g., Leauthaud et al. 2012) - the group finder can yield biases when assessing the halo masses of groups with similar $M_{*}$ values for the central galaxy (Campbell et al. 2015). The $L_{\text {sat }}$ method is optimal at differentiating halo masses for these types of samples.

Do the two AGN samples reside in different halos? In a strict interpretation of that question, WISE-selected AGN live in halos roughly twice as massive as BPT-selected AGN. The distribution of $M_{*}$ for both AGN samples peaks at $10^{10.8} \mathrm{M}_{\odot}$. At this stellar mass, the mean halo mass is $\sim 10^{12.2} h^{-1} \mathrm{M}_{\odot}$ (cf., the compilation of SHMR results in Figure 2 of Wechsler \& Tinker 2018). At this halo mass scale, $L_{\text {sat }}$ corresponds nearly linearly to $M_{h}$. Thus, under the interpretation that the differences in $L_{\text {sat }}$ are driven by changes in $M_{h}$, the $\bar{\chi}$ results from Figure 3 imply halo masses for the two AGN samples of $\log M_{\mathrm{WISE}}=12.58 \pm 0.11$ and $\log M_{\mathrm{BPT}}=12.31 \pm 0.04$. The difference between the two halo masses, $0.27 \pm 0.12 \mathrm{dex}$, is consistent within $1 \sigma$ with the results of DiPompeo et al. (2017) who find $\Delta \log M_{h}=$ $0.45 \pm 0.13$ dex

However, half of the difference in $M_{h}$ between the two
AGN samples can be explained by differences in the host galaxy population. In Figure 2, the stellar mass and color distributions of the two AGN populations are similar, but the $\mathrm{D}_{n} 4000$ values are clearly distinct. Most BPT AGN reside in the transition region between star-forming and quiescent populations. Given the limited statistics of the WISE sample, once the differences $\mathrm{D}_{n} 4000$ are controlled, we cannot rule out the null hypothesis that the difference in $L_{\text {sat }}$ values is driven entirely by the difference in host galaxy properties.

The host galaxy properties themselves offer additional insight. If orientation angle is the only thing that separates dusty AGN from the rest of the population, then the properties of their host galaxies should be the same. Now, the $\mathrm{D}_{n} 4000$ distributions in Figure 2 by themselves are not indication of a fundamental difference in the two populations of AGN; rather, it may indicate selection bias in the construction of the samples. Mendez et al. (2016) also find that IR-selected AGN have a bias toward star-forming galaxies. Kauffmann et al. (2003) demonstrated that the AGN luminosity, quantified through the luminosity on the [OIII] emission line, correlates with $\mathrm{D}_{n} 4000$ such that stronger AGN have younger stellar populations. The left-hand side of Figure 7 shows the distributions of $\log L_{[\mathrm{OIII}]}$ in the BPT sample and the subsample that are also contained within the WISE selection box in Figure 1. Dusty AGN are much stronger AGN than the typical population. Does this account for the differences in their $\mathrm{D}_{n} 4000$ values? The right-hand side of Figure 7 shows that the AGN luminosity accounts for part of the difference, but cannot fully explain the difference in the host galaxy distributions. The red and blue histograms reproduce the $\mathrm{D}_{n} 4000$ distributions from Figure 2. The solid curve shows the distribution for the BPT sample, weighted to reproduce the distribution of $\log L_{[\mathrm{OIII}]}$ for the WISE subsample. Although controlling for luminosity brings the $\mathrm{D}_{n} 4000$ distributions in closer agreement-just as controlling for $\mathrm{D}_{n} 4000$ brings the $L_{\text {sat }}$ values closer - they are still clearly distinct distributions in $\mathrm{D}_{n} 4000$. 
Future low-redshift data will aide in addressing these questions. The Bright Galaxy Survey of the Dark Energy Spectroscopic Instrument (DESI-BGS; DESI Collaboration et al. 2016) will produce an $r$-band limited spectroscopic survey complete to $r=19.5$, roughly two magnitudes fainter than the MGS. This will yield a sample similar in characteristics to the MGS, but larger by volume and number by a factor of 20. This will increase the statistics such that the mean $L_{\text {sat }}-M_{*}$ relation, as in Figure 3, will be a highly discriminating diagnostic. Measurements of $L_{\text {sat }}$ as a function of host halo and AGN properties will provide the leverage necessary to better understand the relationship between halo growth, galaxy growth, and growth of their supermassive black holes in the local universe.

\section{ACKNOWLEDGEMENTS}

The Legacy Surveys consist of three individual and complementary projects: the Dark Energy Camera Legacy Survey (DECaLS; NOAO Proposal ID \# 2014B-0404; PIs: David Schlegel and Arjun Dey), the Beijing-Arizona Sky Survey (BASS; NOAO Proposal ID \# 2015A-0801; PIs: Zhou Xu and Xiaohui Fan), and the Mayall z-band Legacy Survey (MzLS; NOAO Proposal ID \# 2016A-0453; PI: Arjun Dey). DECaLS, BASS and MzLS together include data obtained, respectively, at the Blanco telescope, Cerro Tololo InterAmerican Observatory, National Optical Astronomy Observatory (NOAO); the Bok telescope, Steward Observatory, University of Arizona; and the Mayall telescope, Kitt Peak National Observatory, NOAO. The Legacy Surveys project is honored to be permitted to conduct astronomical research on Iolkam Du'ag (Kitt Peak), a mountain with particular significance to the Tohono O'odham Nation.

NOAO is operated by the Association of Universities for Research in Astronomy (AURA) under a cooperative agreement with the National Science Foundation.

This project used data obtained with the Dark Energy Camera (DECam), which was constructed by the Dark Energy Survey (DES) collaboration. Funding for the DES Projects has been provided by the U.S. Department of Energy, the U.S. National Science Foundation, the Ministry of Science and Education of Spain, the Science and Technology Facilities Council of the United Kingdom, the Higher Education Funding Council for England, the National Center for Supercomputing Applications at the University of Illinois at Urbana-Champaign, the Kavli Institute of Cosmological Physics at the University of Chicago, Center for Cosmology and Astro-Particle Physics at the Ohio State University, the Mitchell Institute for Fundamental Physics and Astronomy at Texas A\&M University, Financiadora de Estudos e Projetos, Fundacao Carlos Chagas Filho de Amparo, Financiadora de Estudos e Projetos, Fundacao Carlos Chagas Filho de Amparo a Pesquisa do Estado do Rio de Janeiro, Conselho Nacional de Desenvolvimento Cientifico e Tecnologico and the Ministerio da Ciencia, Tecnologia e Inovacao, the Deutsche Forschungsgemeinschaft and the Collaborating Institutions in the Dark Energy Survey. The Collaborating Institutions are Argonne National Laboratory, the University of California at Santa Cruz, the University of Cambridge, Centro de Investigaciones Energeticas, Medioambientales y Tecnologicas-Madrid, the University of Chicago,
University College London, the DES-Brazil Consortium, the University of Edinburgh, the Eidgenossische Technische Hochschule (ETH) Zurich, Fermi National Accelerator Laboratory, the University of Illinois at Urbana-Champaign, the Institut de Ciencies de l'Espai (IEEC/CSIC), the Institut de Fisica d'Altes Energies, Lawrence Berkeley National Laboratory, the Ludwig-Maximilians Universitat Munchen and the associated Excellence Cluster Universe, the University of Michigan, the National Optical Astronomy Observatory, the University of Nottingham, the Ohio State University, the University of Pennsylvania, the University of Portsmouth, SLAC National Accelerator Laboratory, Stanford University, the University of Sussex, and Texas A\&M University.

BASS is a key project of the Telescope Access Program (TAP), which has been funded by the National Astronomical Observatories of China, the Chinese Academy of Sciences (the Strategic Priority Research Program "The Emergence of Cosmological Structures" Grant \# XDB09000000), and the Special Fund for Astronomy from the Ministry of Finance. The BASS is also supported by the External Cooperation Program of Chinese Academy of Sciences (Grant \# 114A11KYSB20160057), and Chinese National Natural Science Foundation (Grant \# 11433005).

The Legacy Survey team makes use of data products from the Near-Earth Object Wide-field Infrared Survey Explorer (NEOWISE), which is a project of the Jet Propulsion Laboratory/California Institute of Technology. NEOWISE is funded by the National Aeronautics and Space Administration.

The Legacy Surveys imaging of the DESI footprint is supported by the Director, Office of Science, Office of High Energy Physics of the U.S. Department of Energy under Contract No. DE-AC02-05CH1123, by the National Energy Research Scientific Computing Center, a DOE Office of Science User Facility under the same contract; and by the U.S. National Science Foundation, Division of Astronomical Sciences under Contract No. AST-0950945 to NOAO.

\section{REFERENCES}

Alpaslan M., Tinker J. L., 2019, submitted to MNRAS, arXiv:1911.04509, p. arXiv:1911.04509

Antonucci R., 1993, ARA\&A, 31, 473

Baldwin J. A., Phillips M. M., Terlevich R., 1981, PASP, 93, 5

Blanton M. R., et al., 2005, AJ, 129, 2562

Brinchmann J., Charlot S., White S. D. M., Tremonti C., Kauffmann G., Heckman T., Brinkmann J., 2004, MNRAS, 351, 1151

Campbell D., van den Bosch F. C., Hearin A., Padmanabhan N., Berlind A., Mo H. J., Tinker J., Yang X., 2015, MNRAS, 452, 444

Cappelluti N., Ajello M., Burlon D., Krumpe M., Miyaji T., Bonoli S., Greiner J., 2010, ApJ, 716, L209

Chen Y.-M., et al., 2012, MNRAS, 421, 314

DESI Collaboration et al., 2016, preprint, (arXiv:1611.00036)

Dey A., et al., 2019, AJ, 157, 168

DiPompeo M. A., Myers A. D., Hickox R. C., Geach J. E., Hainline K. N., 2014, MNRAS, 442, 3443

DiPompeo M. A., Hickox R. C., Myers A. D., 2016, MNRAS, 456, 924

DiPompeo M. A., Hickox R. C., Eftekharzadeh S., Myers A. D., 2017, MNRAS, 469, 4630

Donoso E., Yan L., Stern D., Assef R. J., 2014, ApJ, 789, 44 
Hickox R. C., et al., 2011, ApJ, 731, 117

Jarrett T. H., et al., 2011, ApJ, 735, 112

Kauffmann G., et al., 2003, MNRAS, 341, 33

Krumpe M., Miyaji T., Coil A. L., Aceves H., 2018, MNRAS, 474,1773

Lang D., 2014, AJ, 147, 108

Leauthaud A., et al., 2012, ApJ, 746, 95

Li C., Kauffmann G., Jing Y. P., White S. D. M., Börner G., Cheng F. Z., 2006, MNRAS, 368, 21

Mandelbaum R., Wang W., Zu Y., White S., Henriques B., More S., 2016, MNRAS, 457, 3200

McClintock T., et al., 2019, arXiv e-prints, p. arXiv:1907.13167

Mendez A. J., et al., 2016, ApJ, 821, 55

Netzer H., 2015, ARA\&A, 53, 365

Pasquali A., van den Bosch F. C., Mo H. J., Yang X., Somerville R., 2009, MNRAS, 394, 38

Satyapal S., Ellison S. L., McAlpine W., Hickox R. C., Patton D. R., Mendel J. T., 2014, MNRAS, 441, 1297

Sheth R. K., Tormen G., 1999, MNRAS, 308, 119

Strauss M. A., et al., 2002, AJ, 124, 1810

Tinker J. L., Robertson B. E., Kravtsov A. V., Klypin A., Warren M. S., Yepes G., Gottlöber S., 2010, ApJ, 724, 878

Tinker J. L., Hahn C., Mao Y.-Y., Wetzel A. R., Conroy C., 2018, MNRAS, 477, 935

Tinker J. L., Cao J., Alpaslan M., DeRose J., Mao Y.-Y., Wechsler R. H., 2019a, MNRAS, submitted (arXiv:1911.04507), p. arXiv:1911.04507

Tinker J. L., Cao J., Alpaslan M., DeRose J., Mao Y.-Y., Wechsler R. H., 2019b, arXiv e-prints, p. arXiv:1911.04507

Wechsler R. H., Tinker J. L., 2018, ARA\&A, 56, 435

Weston M. E., McIntosh D. H., Brodwin M., Mann J., Cooper A., McConnell A., Nielsen J. L., 2017, MNRAS, 464, 3882

Wright E. L., et al., 2010, AJ, 140, 1868

Yan L., et al., 2013, AJ, 145, 55

Yang X., Mo H. J., van den Bosch F. C., Pasquali A., Li C., Barden M., 2007, ApJ, 671, 153 